\title{
(2) OPEN ACCESS \\ Preclinical models of arthritis for studying immunotherapy and immune tolerance
}

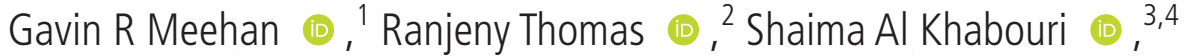 \\ Pascale Wehr, ${ }^{2}$ Catharien MU Hilkens, ${ }^{5}$ David C Wraith, ${ }^{6}$ Daniela Sieghart, ${ }^{7}$

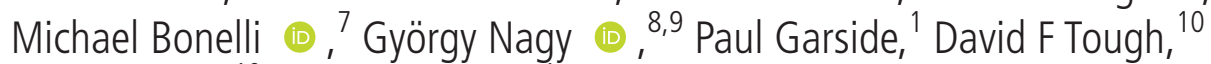 \\ Huw D Lewis, ${ }^{10}$ James M Brewer ${ }^{1}$
}

\begin{abstract}
Handling editor David S
Pisetsky

For numbered affiliations see end of article.
\end{abstract}

\section{Correspondence to} Professor James M Brewer, University of Glasgow, Glasgow G12 8TA, UK

james.brewer@glasgow.ac.uk

Received 1 February 2021 Accepted 27 June 2021 Published Online First 11 August 202

Check for updates

(c) Author(s) (or their employer(s)) 2021. Re-use permitted under CC BY. Published by BMJ.

To cite: Meehan GR,

Thomas R, Al Khabouri S,

et al. Ann Rheum Dis

2021;80:1268-1277.

\section{ABSTRACT}

Increasingly earlier identification of individuals at high risk of rheumatoid arthritis (RA) (eg, with autoantibodies and mild symptoms) improves the feasibility of preventing or curing disease. The use of antigen-specific immunotherapies to reinstate immunological selftolerance represent a highly attractive strategy due to their potential to induce disease resolution, in contrast to existing approaches that require long-term treatment of underlying symptoms.

Preclinical animal models have been used to understand disease mechanisms and to evaluate novel immunotherapeutic approaches. However, models are required to understand critical processes supporting disease development such as the breach of self-tolerance that triggers autoimmunity and the progression from asymptomatic autoimmunity to joint pain and bone loss. These models would also be useful in evaluating the response to treatment in the pre-RA period.

This review proposes that focusing on immune processes contributing to initial disease induction rather than endstage pathological consequences is essential to allow development and evaluation of novel immunotherapies for early intervention. We will describe and critique existing models in arthritis and the broader field of autoimmunity that may fulfil these criteria. We will also identify key gaps in our ability to study these processes in animal models, to highlight where further research should be targeted.

\section{INTRODUCTION}

Rheumatoid arthritis (RA) is a chronic inflammatory autoimmune disease that results in the destruction of the bone and cartilage of the joints. The disease is thought to be driven by genetic predisposition and environmental factors, leading to a loss of immunological self-tolerance, autoimmunity and arthritis (figure 1).

It is widely accepted that the combination of arthralgia and the presence of antibodies (indicating loss of tolerance) to citrullinated proteins (ACPAs) and or IgM rheumatoid factor (RF) is appropriate to identify individuals with high risk of developing RA. ${ }^{1-4}$ Approximately $30 \%-40 \%$ of subjects at risk will develop RA within 1 year. Several factors might indicate even higher risk: (1) high levels of ACPA ( $>$ three times of the upper level of normal) and/ or RF (although RF is probably less important), (2) human leucocyte antigen (HLA) susceptibility alleles, such as shared epitope, (3) evidence of synovitis based on imaging (generally ultrasound and
MRI), (4) smoking and (5) obesity. Based on all these factors individuals with up to $50 \%-60 \%$ risk to develop RA within 1 year might be identified..$^{5-7}$ Disease progression to RA is associated with decreasing potential for remission. ${ }^{8}$ Treatment in the pre-RA phase might be associated with complete suppression of clinical signs and symptoms and the potential for the re-establishment of tolerance. ${ }^{9}$

Current treatments for RA consist of glucocorticoids, conventional and targeted synthetic and biological disease-modifying antirheumatic drugs (DMARDs). However, DMARDs decrease inflammation and ameliorate the radiological progression of the disease without altering the underlying pathology. The focus of recent autoimmune disease research has been to reinstate immunological selftolerance. An 'immunological reset' with antigenspecific immunotherapy may ultimately allow for drug-free remission in RA, in essence curing the disease.

Arthritis research has employed a number of animal models which vary in their design and method of disease induction as well as the stage in the disease process they represent. The benefits of these models and their contributions to research have been discussed extensively in other reviews. ${ }^{10-13}$ Significantly not all models of RA are appropriate for the study of antigen-specific, tolerising immunotherapy.

Here, we focus on models that are suited to the study of initiating events in pre-RA (table 1) and are therefore well placed for identifying therapeutic targets for tolerance induction and for the resulting testing and development of new therapies. Importantly, we identify key questions about arthritis and how these models may contribute to our understanding of different immunological processes and antigen-specific immunotherapies.

\section{Can animal models help us understand loss of tolerance leading to autoimmunity?}

Breach of self-tolerance is a central and early step in the development of autoimmune disease. While the list of self and post-translationally modified antigens that are recognised by the host immune response is increasing, ${ }^{14}$ it remains unclear why responses are directed at these particular proteins, what are the circumstances that drive autoimmune responses to these antigens and why they evade mechanisms of central and peripheral tolerance in RA. Underlying factors associated with RA susceptibility include 


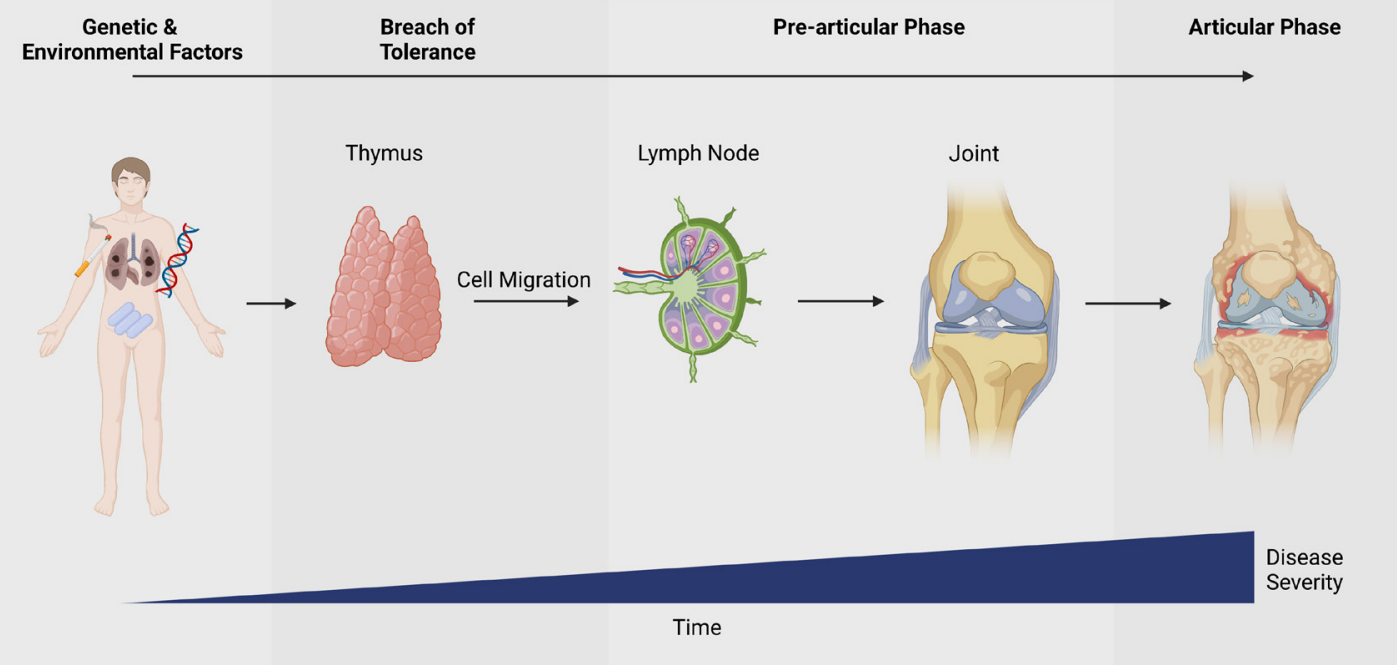

genetic predisposition as well as environmental factors including smoking, various infections, lung inflammation, periodontitis and changes in the microbiome, which contribute to the breach of self-tolerance at mucosal interfaces well before the development of joint inflammation. ${ }^{15-18}$ Animal models can play a critical role in identifying and isolating the environmental and genetic mechanisms that promote loss of tolerance. For example, in animal models with genetic predisposition to autoimmunity, such as the ZAP-70-mutant SKG mouse, in which altered T cell receptor (TCR) signalling leads to modified thymic selection, either an environmental stimulus or additional genetic lesion is required to initiate arthritis. Thus germ-free SKG mice fail to develop peripheral arthritis with a beta-glucan trigger, but they do develop spondylitis. ${ }^{19}{ }^{20}$ SKG mice in a specific pathogenfree environment develop spontaneous arthritis when crossed to ZAP-70-deficient mice. ${ }^{21}$ Equally, models such as collageninduced arthritis (CIA) or proteoglycan (PG)-induced arthritis (PgIA) require specific, susceptible, genetic strains of mice for induction of autoimmunity. ${ }^{22}$ It is worth nothing that while SKG mice have been instrumental for understanding underlying disease mechanisms, they have not been useful to date for studying antigen tolerisation strategies as few self-antigens have been elucidated. ${ }^{23}$

In the CIA or PgIA models, a known antigen is administered to animals in the context of a powerful adjuvant, such as Freund's complete adjuvant or dimethyldioctadecylammonium. This antigen is commonly a heterologous protein that closely resembles the endogenous protein of the animal, although models using autologous antigen have been demonstrated to also effectively induce arthritis in mice. ${ }^{24} 25$ In these models, the adjuvant creates an environment for immunogenicity of the antigen, inducing antibodies cross reacting with heterologous and endogenous antigen, leading to a loss of tolerance. ${ }^{26}$ While these mechanisms are well understood in CIA and PgIA models, they are unlikely to fully reflect how tolerance is breached in patients with RA, which is more complex, without a single initiating autoantigen with adjuvant, and involving the need for an ageing immune system to balance self-tolerance with immune control of micro-organisms. Other models of antigen-induced arthritis (AIA) using molecularly distinct antigens may help answer these questions (figure 2). In ovalbumin (OVA)-induced arthritis (OIA) or AIA, the eliciting antigen (OVA or methylated bovine serum albumin, respectively) is not an autoantigen; however, breach of self-tolerance occurs. This is instigated through the intra-articular injection of antigen into mice previously immunised with the same antigen and may employ the use of adoptively transferred antigen-specific $\mathrm{T}$ cells as in the OIA model. Following this challenge, there is a large influx of neutrophils and macrophages into the joint, resulting in the generation of $\mathrm{B}$ and $\mathrm{T}$ cells that recognise a range of unrelated autoantigens in addition to the initiating antigen (bystander activation). ${ }^{27} 28$ These latter two models allow closer analysis of the conditions that lead to autoimmunity as the bystander response to autoantigen can be considered 'spontaneous'. Using this approach, the key role of cognate antigen (OVA) recognition in the joint and surrounding tissue was identified. Administration of either an inflammatory agent alone (lipopolysaccharides) or OVA subcutaneously is not sufficient to elicit autoimmunity. ${ }^{29}{ }^{30}$ Further studies defined the role played by endogenous conventional dendritic cells (DC) in promoting breach of tolerance, as well as the regulatory role of plasmacytoid DCs. ${ }^{31}{ }^{32}$ Future studies of these models will help define the range of autoantigens that are recognised in joint inflammation and, more importantly, when and why these particular host antigens are recognised and how they promote the process of epitope spreading. In this respect, it is important to note that immune recognition of post-translational modifications of endogenous proteins such as citrullination have been observed at low levels in some models, ${ }^{29}$ although there have been questions about the reproducibility of these results as well as the absence of appropriate controls. ${ }^{33}$ Whether ACPA are directly pathogenic in RA is still unclear. However, studies aimed at tolerising the $\mathrm{T}$ cell response to citrullinated antigens in both animals and humans may help define whether regulation of this response influences disease outcome.

While some models above contribute to our understanding of why breach of tolerance and autoimmunity develops, there remains considerable scope for improvement. Animal models offer the opportunity to perform reductionist approaches that allow dissection of the complex contributory genetic and environmental factors that lead to breach of tolerance. However, the mechanisms driving disease events in animal models do not necessarily replicate those occurring in human RA, for example, respiratory mucosal involvement, complex genetic background and contributory environmental factors, in addition to the long duration of disease. Furthermore, no spontaneous models faithfully reproduce human RA. Technologies such as animals 


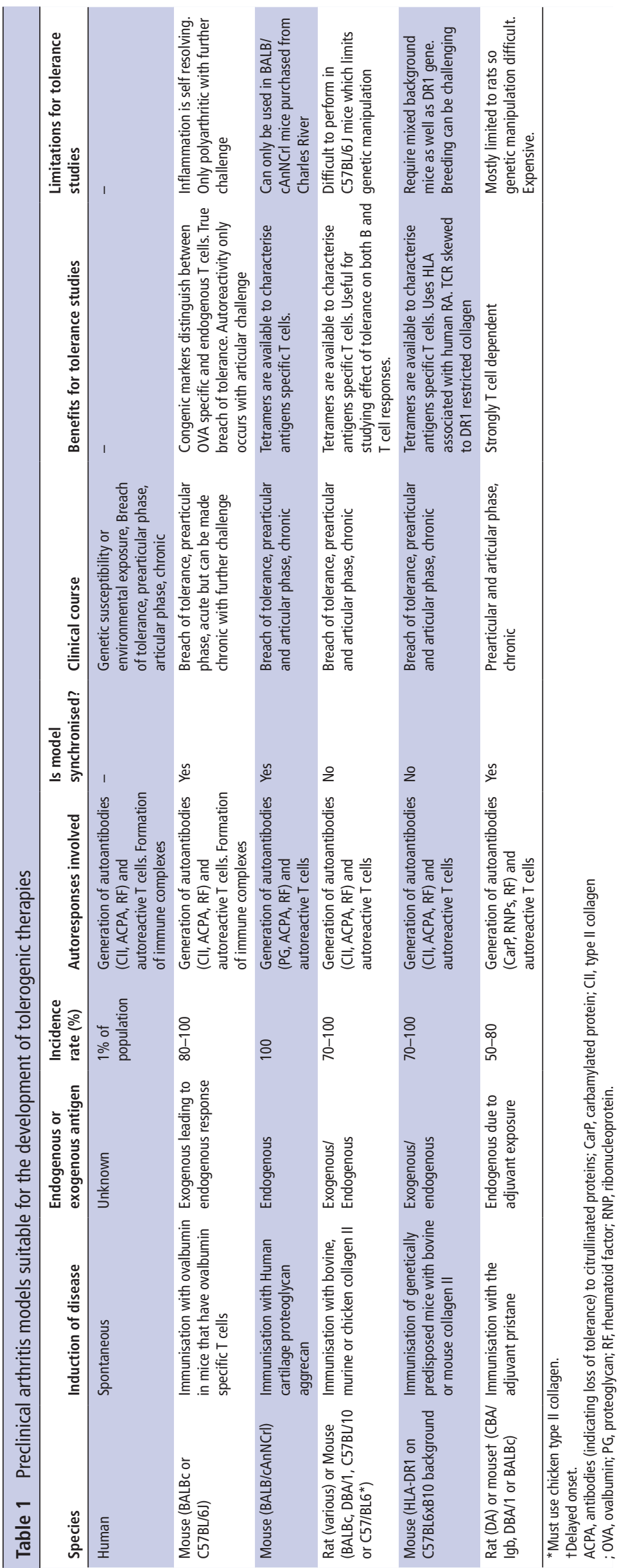


(i)

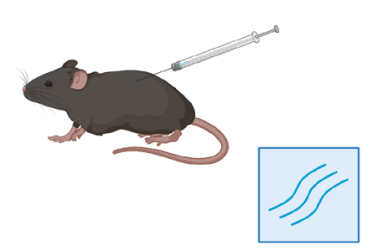

(ii)

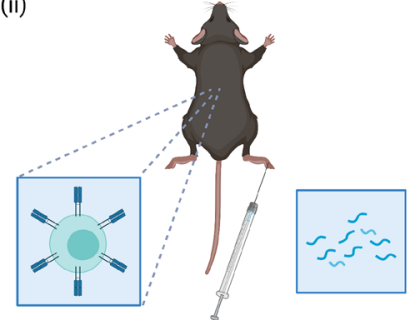

(iii)

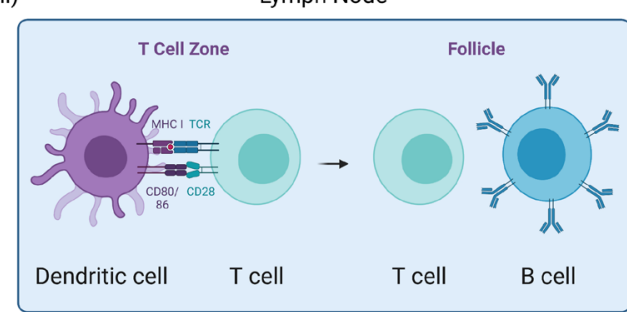

(iv)

Cytokines

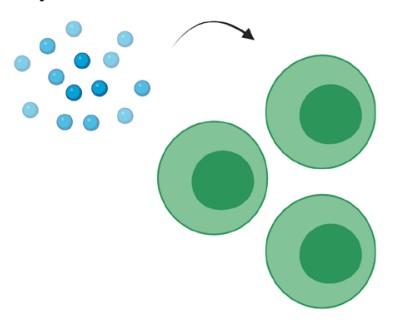

Bystander T Cell Activation (v)

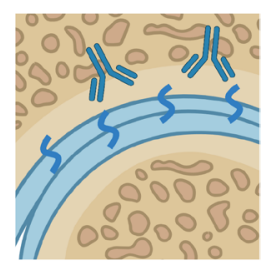

(vi)

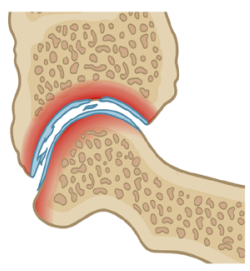

Figure $2 \mathrm{CIA}$ and AIA models of arthritis. (i) CIA mice are injected with heterologous or autologous collagen in the presence of an adjuvant. (ii) in AIA models, mice are first immunised with an unrelated antigen in the presence of an adjuvant and then rechallenged with the same antigen in the joint. These models may employ the use of TCR transgenic T cells. (iii) The antigens in both models are initially presented by dendritic cells to CD4 T cells within the T cell zone of the lymph nodes. These CD4 T cells then interact with B cells within the follicle to produce antibodies. (iv) In the AIA models, the inflammation within the joint to the exogenous antigen triggers the activation of bystander $T$ cells resulting in the targeting of joint antigens. (v) In both models, antigens within the joints become targeted by the immune response. (vi) This results in the destruction of cartilage and bones within the joints - created with BioRender.com. AIA, antigen-induced arthritis; CIA, collagen-induced arthritis; TCR, T cell receptor

expressing fluorescent reporters can be used to identify where and when key molecules are expressed, while cell-specific and tissue-specific gene knockouts can identify their mechanistic contributions to autoimmunity. These studies can be performed with the opportunity for the full temporal development of autoimmunity to be investigated, including assessment of where and when key therapeutic windows arise.

\section{Can animal models help us understand the progression from asymptomatic autoimmunity to joint infiltration and bone erosion?}

The development of autoimmunity in RA and the transition into clinical disease remains a poorly understood process. Changes in innate immune reactivity and altered $\mathrm{T}$ cell and $\mathrm{B}$ cell regulation result in the development of autoantibodies targeting post-translationally modified proteins. These perturbations in immune cell activity indicate loss of tolerance and eventually culminate in the development of a synovial lesion that contains large numbers of infiltrating $\mathrm{T}$ cells, $\mathrm{B}$ cells, macrophages and fibroblasts. $^{34}$

As this transitionary period generally occurs slowly over many years, different aspects of the immune response, particularly within the joints and lymph nodes, are difficult to study longitudinally in patients. Although animal models are unable to fully recapitulate human disease, their selective application has offered many insights into the development of autoimmunity and the complex interplay of immune cells in different tissues at various stages of disease. Importantly, as these models can be used in combination with technologies that would be otherwise impractical or unethical for use in patients, they allow for the study of discrete aspects of the disease that cannot be researched using other methods.

The ability to identify, manipulate and track specific cell populations is particularly useful in animal models, as has been shown in research examining the roles of autoreactive CD4 T cells in the development of early arthritis. The PgIA model has been used to demonstrate that TCR signalling strength dictates the fate of $\mathrm{T}$ cells, with those with weaker signals developing into $\mathrm{T}$ follicular helper cells (Tfh) which stimulate human PG-specific antibodies, cross-reactive with mouse PG. ${ }^{35}$ Since autoreactive $\mathrm{T}$ cells driving autoimmunity may have escaped central and peripheral tolerance mechanisms due to low TCR affinity, the fact that autoreactive $\mathrm{T}$ cells in RA mostly recognise modified self, which bind HLA with higher affinity, offers insights into the activation and persistence of Tfh and other effector cells driving autoimmune disease progression.

$\mathrm{T}$ cell migration studies, using multiphoton microscopy and lymph node sequestering drugs ${ }^{36}$ have also demonstrated that the majority of aggrecan-specific $\mathrm{T}$ cells are not involved in the pathogenesis of synovial inflammation directly, but rather exert their effects in the lymphoid organs where they provide B cell help for systemic autoantibody production. ${ }^{37-39}$ Similar work using a partially humanised CIA model in HLA-DR1 isotype (HLA-DR1) mice, in which chimeric human/mouse major histocompatibility complex (MHC) class II molecules comprise the peptide-binding domain from human DR and the CD4-binding domain derived from mouse I-E, ${ }^{40}$ has shown that $\mathrm{T}$ cells expressing an RA-relevant HLA-class II allele mount a response to the dominant epitope of collagen II. In this model, at the time of first clinical arthritis symptoms, specific effector CD4 T cells were undetectable in the synovial fluid and rare in the blood, but persisted in the lymph nodes. ${ }^{42}$

Taken together, data in PgIA and CIA models suggest that after the initial antigen-specific CD4 + T cell priming event in the lymphoid organs, disease development is dependent on $\mathrm{B}$ cells, which can present antigen and produce antibodies, and is perpetuated by CD4 Tfh cells which provide further B cell help for antibody-mediated joint destruction. ${ }^{43}{ }^{44}$ Methods that disrupt Tfh and $\mathrm{B}$ cells within the lymph node may therefore offer a potential target for new immunotherapies. 
Aside from T cells, animal models also implicate many other immune cells in arthritic disease development and regulation, including $\mathrm{B}$ cells, ${ }^{45}$ plasmacytoid $\mathrm{DCs}^{31}$ and synovial fibroblasts. ${ }^{46}$ Animal models offer major insights into immune cell dysfunction in arthritis. As new tolerogenic therapies are developed, antigen-driven animal models will be essential tools to understand how treatments impact immunological processes and will be key to understanding how these therapies function to restore immunological tolerance.

\section{How does the diversity of the TCR repertoire influence models?}

TCR repertoire diversity is achieved on two levels: a genetic level involving selecting, editing and combining the various TCR genes, and on a cellular level involving thymic selection and outgrowth of certain clones in both acute and chronic immune responses. The strong association of autoimmune diseases, including RA, with certain HLA alleles is well documented. ${ }^{47-49}$ Thus, it is plausible that thymic selection and peripheral antigen encounter could influence the composition of the mature $T$ cell repertoire in persons susceptible to RA and in patients with RA. ${ }^{51}$ Indeed, the outgrowth or enrichment of certain $\mathrm{T}$ cell clones has been demonstrated in RA, both in the naïve ${ }^{52}$ and antigen-experienced $\mathrm{T}$ cell compartments ${ }^{53-56}$ suggesting that both thymic selection and antigen-driven responses skew the TCR repertoire in patients with RA. Similarly in the CIA model in DBA/ 1 mice, the $I A q$ allele is required for development of the disease due to high affinity binding of the collagen II dominant epitope to I-Aq after processing of collagen II protein, driving activation of autoreactive T cells. ${ }^{5758}$

Moreover, TCR repertoire diversity in patients with RA differs depending on the tissues sampled. For instance, the repertoire was found to be more restricted in the synovial compartment compared with peripheral blood in patients with RA, ${ }^{535560}$ indicating that tissue sites may influence the retention or accumulation of CD4 $\mathrm{T}$ cells possibly in an antigenspecific manner. TCR diversity has also been found to evolve with RA chronicity. In some cases, the TCR repertoire was more restricted in early RA and diversified with the progression of the disease, ${ }^{54}$ while in other cases the TCR repertoire was found to become more restricted with time. ${ }^{61}$ Additionally, changes in the TCR repertoire can also indicate patient response to therapeutics. For instance, patients treated with tumour necrosis factor inhibitors showed a reduction in clonal expansion in $\mathrm{T}$ cells expressing certain TCR $\beta$ variable region (TCRBV) genes, ${ }^{62}$ while responders and non-responders to methotrexate display differences in TCRBV gene expression profiles in the circulating CD4 T cell repertoire. ${ }^{63}$

The differences in TCR repertoire diversity reported at various stages of RA development and between different tissue sites highlights how assessment of TCR repertoire diversity has the potential of being an informative indicator of disease state and predictor of effective therapeutic regimens. However, patient to patient variability in clonal responses and the conflicting evidence of repertoire changes with disease progression accentuate our lack of understanding of how TCR repertoire diversity develops in RA and how it evolves with disease progression. Thus, animal models of arthritis can help elucidate development of the TCR repertoire as they provide a setting in which different disease stages can be observed more easily and allow for spatial and temporal assessment of TCR diversity. ${ }^{62}{ }^{63}$ In addition, mouse models, such as CIA, with known dominant epitope, restricting I-A and HLA-DR molecules and responding T cells that can be identified with pMHC tetramers, offer a major advantage for T cell tolerance studies.

Models already exist that incorporate the influence of thymic selection on susceptibility to develop arthritis. For example, C57BL/6N.Q mice are more susceptible to CIA compared with $\mathrm{C} 57 \mathrm{BL} / 6$ mice due to differences in MHC restriction ${ }^{64} 65$ and changes in $\mathrm{T}$ cell positive and negative selection in the SKG transgenic mice result in spontaneous development of arthritis. ${ }^{23}$ Studies examining aspects of TCR repertoire diversity have been conducted using the CIA model of arthritis and have also reported a skewed or restricted TCR repertoire and the prevalence of certain TCR $\beta$ chains were found to be strain dependent. $^{66-69}$ The dominance of these chains were also relevant to the pathology as administration of depleting antibodies specific to the dominant $\mathrm{V} \beta$ chains were found to significantly reduce the incidence of CIA. One study using the HLA-DR1 mouse/CIA model found CD4 T cells of limited clonality in the joint with a highly selective subset of the TCR repertoire. ${ }^{70}$ These CD4 $\mathrm{T}$ cells bind to the dominant collagen II epitope and, although they comprise a minor population, they may play a major role in disease pathogenesis. A recent study investigated differences in the composition of the TCR repertoire in joints and their draining lymph nodes with the progression of OIA. ${ }^{71}$ The authors reported a disparity in TCR repertoire diversity between the draining lymph nodes and joints with the progression of inflammatory arthritis, with the lymph nodes displaying greater repertoire diversity than the joints at later stages of the disease. The results of the study highlight two main therapeutic implications; first, that tolerogenic therapies may be more effective at the very early stages of arthritis when the TCR repertoire is more restricted and, second, that TCR repertoire of joint-draining lymph nodes could possibly foreshadow TCR repertoire diversity of the joint, and thus be a marker of disease severity and guide effective therapeutic interventions. Significantly, animal models provide the opportunity to test these hypotheses, and rationalise the application of antigen-specific immunotherapy in disease.

\section{Are particular models more suitable for studying specific immunotherapeutic approaches?}

There is a wide range of animal models available for arthritis research but not all models are well suited for studying tolerogenic immunotherapies. As these therapies can take many different forms it is essential that models are selected with consideration given to the method of tolerance induction. Optimising model selection will strengthen the data garnered from these studies and should improve the translation of this research into effective clinical treatments.

In the pathogenesis of RA, DCs act as key players in the development of autoimmunity as they, along with medullary thymic epithelial cells, present self-antigens to $\mathrm{T}$ cells in the thymus impacting negative selection, and in the periphery they are able to prime naive autoreactive T cells to initiate autoimmune models. ${ }^{72}$ However, DCs are also capable of inducing and maintaining peripheral tolerance by blocking $\mathrm{T}$ cell expansion, inducing $\mathrm{T}$ cell deletion or anergy. One promising cell-based approach is targeting autoreactive $\mathrm{T}$ lymphocytes by the production of tolerogenic DCs (TolDCs). The tolerogenic function of DCs can be promoted by the exposure to different anti-inflammatory cytokines or by in vitro treatment with an NF-kB inhibitor. TolDCs act by different mechanisms including the secretion of immunomodulatory mediators, reduction of MHC and costimulatory molecules or the expression of immune-modulatory/ 
immune-inhibitory molecules. ${ }^{73}$ Preclinical data informing current clinical trials of TolDC immunotherapy in RA were derived from the 'classical' RA models, namely CIA ${ }^{74-76}$ and AIA models. ${ }^{77}$ Humanised mouse models of RA show several advantages in testing tolerogenic therapy by enabling direct translation to humans through introduction of human transgenes or by the selective transfer of human autoantigens or cells/tissue into immunodeficient mice. ${ }^{78}$ However, limitations include relatively poor expression of the human HLA transgene, and the need for induction of inflammatory arthritis with heterologous antigen, which limit interpretation of antigen presentation and efficacy of tolerising immunotherapies. ${ }^{79}$

The induction of regulatory $\mathrm{T}$ cells (Treg) by peptide-based therapies have been developed for the treatment of a number of autoimmune diseases including RA, ${ }^{80}$ multiple sclerosis (MS) and Graves' disease. ${ }^{81-83}$ In this treatment, known tolerogenic peptides bind directly to MHC II on DCs. ${ }^{84}$ These DCs then interact with CD4 $\mathrm{T}$ cells to induce regulatory $\mathrm{T}$ cells that suppress $\mathrm{T}$ cell activation. As this therapy is based on peptide presentation, HLA-DR transgenic mice have supported the design of tolerogenic $\mathrm{T}$ cell epitopes and testing of tolerogenic strategies ${ }^{85-87}$; however, important lessons have been learnt. For example, introduction of a human HLA allele does not guarantee that an HLA-DR transgenic mouse will respond to an epitope known to be dominant in humans. ${ }^{88}$ This implies that mice have a 'hole' in their T cell repertoire for certain HLArestricted $\mathrm{T}$ cell epitopes which can be overcome by creation of mice expressing both HLA-DR and TCR molecules from relevant patients. ${ }^{85}{ }^{89}$ Furthermore, design work with individual peptide epitopes has shown that they must mimic naturally processed epitopes when bound to MHC II in order to induce tolerance through induction of IL-10 secreting regulatory $\mathrm{T}$ cells. ${ }^{90} 91$ This research confirms the importance of HLA-DR mice for the development and testing of peptide-based therapies in RA.

In addition to antigen-specific immunomodulatory therapy targeting DCs or T cells in situ, chimeric antigen receptor (CAR)Treg cell therapy, in which Tregs are engineered to target specific proteins in a MHC independent manner, ${ }^{92} 93$ is being expanded to include autoimmunity in light of promising results from clinical trials, and product registration of CAR-T in oncology. ${ }^{94}$ In the context of RA and the HLA-DR1 model, it has been reasoned that engineering CAR-Tregs to specifically target an antigen in the joints of patients with RA may promote their migration to the site of abnormal inflammation, inducing a localised and protective immunosuppressive response. Accordingly, a CAR directed against citrullinated vimentin, a cytoskeletal protein, which is expressed in the synovial tissue of the majority of patients with RA, has been developed. ${ }^{95}$ This group is working to transduce this CAR into Tregs in order to assess functional activity in vitro and therapeutic potential in vivo of CAR-Treg transfer in the CIA model. Another approach in development is the generation of CAR CD8 CTL presenting antigenic peptide to specifically target and eliminate autoreactive CD4 T cells (Rosloniec, unpublished); these will also be tested in the HLADR1 humanised mouse model of CIA. While the CAR-Treg approach is advantageous in that it offers specific targeting and imparts no HLA restriction, its drawback is the requirement for a specific antigen for recognition, which is a design issue in RA due to the number of potential autoantigens involved in disease progression. Strategies invoking bystander tolerance or patient stratification based on putative autoantigen involvement and disease stage may facilitate therapeutic selection of CAR-T cell therapy to complement immunomodulatory approaches such as antigen-specific immunotherapies, as have been used in solid tumours in vivo in mice ${ }^{96}$

One of the oldest and most widely explored tolerogenic therapies is antigen feeding. In this therapy, small amounts of a specific antigen are administered orally to restore a state of homeostasis and tolerance to self-peptides in the adaptive immune system. This method has been used extensively with antigen-induced models, particularly CIA. Multiple experiments demonstrated that feeding collagen II prior to disease induction was protective against CIA in rats. ${ }^{97} 98$ Unfortunately, subsequent clinical trials with patients with RA showed conflicting results, ${ }^{99-101}$ with greater success observed with administration of lower antigen doses leading to the generation of active suppression via Tregs rather than anergy or clonal deletion. ${ }^{102}$ Due to inconsistencies between trials, this therapy was not pursued in RA. The disparity between animal models and clinical studies may lie in the lack of knowledge about the initiating autoantigen in RA, as collagen II is just one of many possible autoantigens involved in disease progression. Similarly, the timing of clinical trials of antigen feeding may be too late when autoimmunity has already progressed to disease. In addition, differences in rodent and human immune responses have to be considered. ${ }^{103}$ Despite these setbacks, antigen-induced CIA, OIA and AIA models are certainly useful to understand the mechanisms of how tolerance is induced from an immunological perspective. They may also offer insights into how antigen dosing and the timing of intervention affects disease outcomes.

DC targeting with antigen in the context of suppressing their activation is an emerging immunotherapy that is gaining popularity. DC targeting recapitulates models in which transgenic antigen targeted to 'resting' DCs promotes long-lasting peripheral tolerance through mechanisms of $\mathrm{T}$ cell deletion or regulation. ${ }^{104}$ Nanoparticles such as liposomes encapsulating disease-specific peptides along with immunomodulatory drugs, such as curcumin or calcitriol to suppress NF-kB activation required for DC activation, are taken up by DCs that interact with antigen-specific CD4 T cells to suppress disease progression. ${ }^{105}$ In the PgIA model, tolerising liposomes were found to significantly suppress disease severity. ${ }^{106}$ Peptide/calcitriol liposomes were found to exert their effects primarily through the deletion of high affinity antigen-specific autoreactive CD4 T cells and through anergy induction in the residual antigen-specific T cells. Delivery of the tolerising liposomes after the onset of disease also significantly reduced disease severity, even though arthritis is predominantly driven by autoantibody and complement-driven mechanisms in established disease. ${ }^{107}$ In contrast to pretreatment, the liposomes in this experiment were found to exert their effects through the expansion of FoxP3 + and IL-10-producing Tregs. Interestingly, this model suggests that the mechanisms of tolerance induction are dependent on the timing of liposome administration.

\section{Will current animal models identify where and when to intervene?}

One of the major strengths of animal models of RA is that they allow for in-depth investigation of molecular and cellular processes at all different disease stages, that is, from initiation to chronic inflammation. They, therefore, also provide a powerful tool for studying immunotherapies, addressing important questions relating to the timing, route and frequency of administration and therapeutic effects. For example, using a rat allotransplantation model it was found that the timing and frequency of mesenchymal stem cell administration was crucial for graft survival, with multiple administrations having the best 
(i)

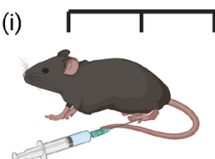

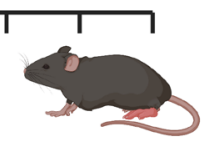

(ii)

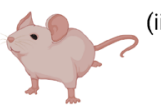

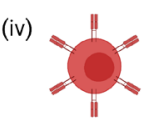

(iii)

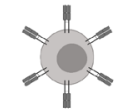

(B) Interventions

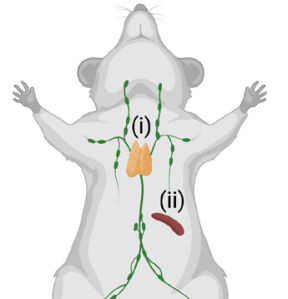

(iii)
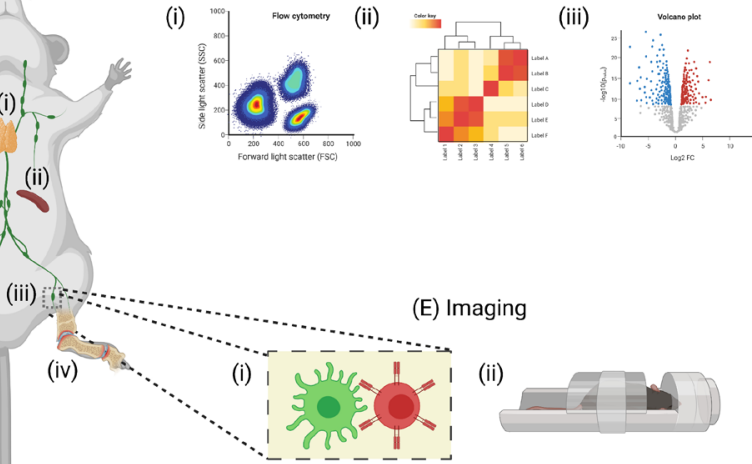

(E) Imaging

(ii)

(iii)

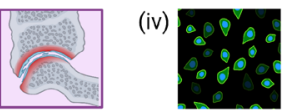

Figure 3 Benefits of using animal models for studying rheumatoid arthritis. Animal models allow researchers to study various aspects the disease that would otherwise be impractical to study in human patients. (A)(i)The experimental design of animal models allow researchers to monitor disease progression at various time points. Specific aspects of the disease can also be examined through the use of (ii) transgenic animals, (iii) TCR transgenic T cells and (iv) fluorescently labelled cells. (B) Interventions including (i) antigen-specific immunotherapies and (ii) drug treatments can also be studied in detail. (C) Tissues including the (i) thymus, (ii) spleen, (iii) lymph nodes and (iv) synovial tissue can be collected from animals at any time point. (D) This allows for detailed analysis of various cell populations using techniques such as (i) flow cytometry, (ii) RNA sequencing and (iii) cytokine assays. (E) Another major advantage of animal models is the use of live imaging techniques including (i) intravital imaging using multiphoton microscopy and (ii) whole tissue imaging using techniques such as MRI scanners. Similarly, tissues collected from culled animals can be imaged by (iii) histology or (iv) immunofluorescence - created with BioRender.com.

outcome in terms of the number of circulating Tregs. ${ }^{108}$ Similarly, administration of IL-4-transduced DC in CIA mice via the intravenous or intraperitoneal routes led to higher numbers of DC migration to the spleen and correlated with enhanced therapeutic effects as compared with the subcutaneous administration route. ${ }^{109}$

The disease stage is particularly important for immunomodulatory tolerance induction strategies, which use Tregs. The function, survival and stability of these cells is highly influenced by inflammation and tissue-specific factors which will vary depending on the stage and activity of the disease. ${ }^{110}$ Functional adaptation of FoxP3 + Tregs, also referred to as Treg plasticity, is an important process that occurs during protective immune responses. For example, exposure of Tregs to polarising cytokines directs expression of appropriate chemokine receptors that allow Tregs to home to and regulate the relevant site of inflammation. However, chronic exposure of Tregs to inflammatory mediators, as might occur, for example, in active RA, can backfire by destabilising FoxP3 expression and turn Tregs into pathogenic effector T cells. Indeed, it was shown that synovial fibroblastderived IL-6 converted FoxP3 Tregs into Th17 cells with potent osteoclastogenic function in a CIA mouse model. ${ }^{111}$ This has important implications for Treg-based therapies, whether it is through adoptive transfer of Tregs, induction of FoxP3 + Tregs via adoptive transfer of tolerogenic DCs or in vivo expansion of existing Tregs with low dose IL-2, which shows promise in lupus as well as other autoimmune diseases. ${ }^{112}$ To avoid a detrimental conversion of Tregs, further investigation is required to optimise the timing of administration of tolerogenic immunotherapies, the potential for coadministration of anti-inflammatory drugs that could prevent Treg conversion (eg, anti-IL-6), and strategies and conditions that support or induce stable type $1(\operatorname{Tr} 1)$ Treg from memory $\mathrm{T}$ cells.

Conversely, it is important to consider potentially adverse effects of existing RA medications on tolerance induction.
For example, mouse models have shown that the calcineurin inhibitor ciclosporin A interferes with induction of allograft tolerance, ${ }^{113}$ and Cox-2 inhibitors (a subclass of non-steroidal anti-inflammatory drugs) inhibit oral tolerance to dietary antigens. ${ }^{114}$ The inhibitory effect of ciclosporin $\mathrm{A}$ is most likely caused by inhibition of Treg expansion and function. ${ }^{115-117}$ Testing the in vivo effects of relevant RA drugs on performance of tolerogenic therapeutics in preclinical animal models is important to determine the most suitable patient group for recruitment to clinical trials, and which DMARDs might help or hinder the tolerogenic response.

Another important question is where protolerogenic therapies should act. There is ample evidence that peripheral tolerance is chiefly induced in secondary lymphoid tissues-the same site as for priming of tissue-specific $\mathrm{T}$ cell clones. For example, immune tolerance to inhaled or oral antigens relied on CCR7-dependent migration of DCs to the relevant draining lymph nodes, ${ }^{118} 119$ and induction of allograft tolerance through treatment with IL-10-producing DCs also depended on CCR7mediated homing of these DCs to the lymph node. ${ }^{120}$ It is not surprising that secondary lymphoid tissues play an important role in both immunogenic and tolerogenic immune responses, given that DCs (both mature and immature 'tolerogenic' DCs) as well as naïve $\mathrm{T}$ cells and Tregs home to these locations, providing the optimal architecture relevant for DC/T cell interactions. However, it is still uncertain whether this precludes the possibility that tolerance could be induced in different locations, for example, ectopic lymphoid structures at sites of inflammation (eg, in the rheumatoid joint) as with infiltrating Tregs that control tissue-destructive tumour-infiltrating lymphocytes in tumour sites. ${ }^{121}$ Understanding at which sites tolerance induction is most effective or even possible is critical to determine and to develop technologies for the most optimal routes of tolerogenic antigen (eg, TolDC) administration. Addressing these questions in humans is a major challenge. Although studies are 
underway to compare different routes of TolDC administration (intradermal vs intranodal) in the RESTORE study in patients with MS, ${ }^{122}$ and intradermal versus intra-articular versus intranodal in the AuToDeCRA2 study in patients with RA (Isaacs and Hilkens, unpublished), partially humanised animal models could aid in investigating these questions in more depth. For example, animal models provide an excellent tool for the longitudinal tracking and visualisation of interactions between different cell populations in vivo, including PET combined with vascular or lymphotracking dyes and CT or MRI, as well as multicolour fluorescence imaging. In some circumstances, these can be translated to clinical trials. Animal models can therefore be hugely beneficial in getting important clues on when and where to intervene, allowing for the improved, informed design of future clinical trials in patients with RA.

\section{CONCLUSION}

Although there have been many criticisms of animal models due to the poor translatability of data from preclinical models to clinical trials, ${ }^{123}$ currently these models remain essential to develop curative therapy in RA. Understandably not all aspects of human disease can be fully recapitulated in animal models including the long transition from breach of tolerance to autoimmunity as well as the extensive interplay of genetic and environmental factors that trigger the onset of disease. Despite these drawbacks, when proficiently applied in combination with different technologies, and selected to reflect appropriate points in disease progression, animal models are critical tools in mechanistic arthritis research and remain essential for the development of curative therapies (figure 3).

A key point is that of reverse translation. As new antigenspecific immunotherapies are developed, it is critical that data from clinical studies further inform model selection. This will allow for a targeted approach to research in animal models, where bioassays or technologies can be improved for future trials, and to identify the immunological mechanisms underlying human disease and therapeutic responses. Used in this way, animal models will facilitate the development and testing of new therapeutic agents to reinstate immunological self-tolerance.

\author{
Author affiliations \\ 'Institute of Infection, Immunity and Inflammation, University of Glasgow, Glasgow, \\ UK \\ ${ }^{2}$ University of Queensland Diamantina Institute, The University of Queensland, \\ Woolloongabba, Queensland, Australia \\ ${ }^{3}$ Division of Rheumatology, Department of Medicine, Karolinska Institutet, Karolinska \\ University Hospital, Stockholm, Sweden \\ ${ }^{4}$ Center for Molecular Medicine, Karolinska University Hospital Solna, Stockholm, \\ Sweden \\ ${ }^{5}$ Translational \& Clinical Research Institute, Newcastle University, Newcastle upon \\ Tyne, UK \\ Institute of Immunology and Immunotherapy, College of Medical and Dental \\ Sciences, University of Birmingham, Birmingham, UK \\ ${ }^{7}$ Division of Rheumatology, Department of Internal Medicine III, Medical University of \\ Vienna, Vienna, Austria \\ ${ }^{8}$ Department of Rheumatology \& Clinical Immunology, Semmelweis University, \\ Budapest, Hungary \\ ${ }^{9}$ Department of Genetics, Cell and Immunobiology, Semmelweis University, \\ Budapest, Hungary \\ ${ }^{10} \mathrm{GlaxoSmithKline} \mathrm{Research} \mathrm{and} \mathrm{Development,} \mathrm{Stevenage,} \mathrm{UK}$
}

Twitter Gavin R Meehan @LIVE_iiiglasgow

Contributors All authors contributed to the design, layout and content of the review. GM wrote and edited the manuscript and prepared the table and figures. RT, SAIK, PW, CMUH, DCW, DS, MB and JMB wrote different sections of the manuscript. GN, PG, DT and HW edited the manuscript.

Funding This project has received funding from the Innovative Medicines Initiative 2 Joint Undertaking under grant agreement No 777357. This Joint Undertaking receives support from the European Union's Horizon 2020 research and innovation programme and EFPIA. www.imi.europa.eu

Disclaimer This communication reflects the authors views and neither IMI nor the European Union, EFPIA, or any Associated Partners are responsible for any use that may be made of the information contained within.

Competing interests GM, RT, CMUH, DCW, DS, MB, PG and JMB all received funding from the Innovative Medicines Initiative 2 Joint Undertaking under grant agreement No 777357. DCW is the founder and serves as a consultant to Apitope International NV. RT reports additional grants from Arthritis Queensland, and an NHMRC senior research fellowship during the conduct of the study; grants from NHMRC grants 1083192 and 1071822, past funding from Janssen Biotech Inc to Uniquest outside the submitted work; In addition, RT has patent 9,017,697 B2: 2006 issued, a grant from JDRF Australia and US The Leona M. and Harry B. Helmsley Charitable Trust for antigen-specific immunotherapy in type 1 diabetes, and investment from CSL to UniQuest to develop and commercialise antigenspecific immunotherapy in Sjogren's syndrome. DS and MB report grants from Medical University of Vienna during the conduct of the study. HDL and DFT are both employees and shareholders of GSK (Pharma partner in RTCure Consortium).

Patient and public involvement Patients and/or the public were not involved in the design, or conduct, or reporting, or dissemination plans of this research.

Patient consent for publication Not required.

Provenance and peer review Not commissioned; externally peer reviewed.

Open access This is an open access article distributed in accordance with the Creative Commons Attribution 4.0 Unported (CC BY 4.0) license, which permits others to copy, redistribute, remix, transform and build upon this work for any purpose, provided the original work is properly cited, a link to the licence is given, and indication of whether changes were made. See: https://creativecommons.org/ licenses/by/4.0/.

\section{ORCID iDs}

Gavin R Meehan http://orcid.org/0000-0001-9855-6565

Ranjeny Thomas http://orcid.org/0000-0002-0518-8386

Shaima Al Khabouri http://orcid.org/0000-0002-5147-9627

Michael Bonelli http://orcid.org/0000-0002-6122-7482

György Nagy http://orcid.org/0000-0003-1198-3228

\section{REFERENCES}

1 Gerlag DM, Raza K, van Baarsen LGM, et al. EULAR recommendations for terminology and research in individuals at risk of rheumatoid arthritis: report from the study Group for risk factors for rheumatoid arthritis. Ann Rheum Dis 2012;71:638-41.

2 van Steenbergen HW, Aletaha D, Beaart-van de Voorde LJJ, et al. EULAR definition of arthralgia suspicious for progression to rheumatoid arthritis. Ann Rheum Dis 2017;76:491-6

3 Ten Brinck RM, van Steenbergen HW, van Delft MAM, et al. The risk of individual autoantibodies, autoantibody combinations and levels for arthritis development in clinically suspect arthralgia. Rheumatology 2017;56:2145-53.

4 Boeters DM, Raza K, van der Helm-van Mil AHM. Which patients presenting with arthralgia eventually develop rheumatoid arthritis? the current state of the art. RMD Open 2017;3:e000479.

5 Ten Brinck RM, van Steenbergen HW, van der Helm-van Mil AHM. Development of clinically apparent synovitis: a longitudinal study at the joint level during progression to inflammatory arthritis. RMD Open 2018;4:e000748.

6 Al-Laith M, Jasenecova M, Abraham S, et al. Arthritis prevention in the pre-clinical phase of RA with abatacept (the APIPPRA study): a multi-centre, randomised, double-blind, parallel-group, placebo-controlled clinical trial protocol. Tria/s 2019;20:429.

7 Bos WH, Wolbink GJ, Boers M, et al. Arthritis development in patients with arthralgia is strongly associated with anti-citrullinated protein antibody status: a prospective cohort study. Ann Rheum Dis 2010;69:490 LP-4.

8 Monti S, Montecucco C, Bugatti S, et al. Rheumatoid arthritis treatment: the earlier the better to prevent joint damage. RMD Open 2015;1:e000057.

9 Nagy G, van Vollenhoven RF. Sustained biologic-free and drug-free remission in rheumatoid arthritis, where are we now? Arthritis Res Ther 2015;17:181.

10 Benson RA, McInnes IB, Garside P, et al. Model answers: rational application of murine models in arthritis research. Eur J Immunol 2018;48:32-8.

11 Vossenaar ER, Nijenhuis S, Helsen MMA, et al. Citrullination of synovial proteins in murine models of rheumatoid arthritis. Arthritis Rheum 2003;48:2489-500.

12 Bendele A. Animal models of rheumatoid arthritis. J Musculoskelet \& neuronal Interact 2001;1:377-85.

13 Asquith DL, Miller AM, McInnes IB, et al. Animal models of rheumatoid arthritis. Eur J Immunol 2009;39:2040-4.

14 Derksen VFAM, Huizinga TWJ, van der Woude D. The role of autoantibodies in the pathophysiology of rheumatoid arthritis. Semin Immunopathol 2017;39:437-46. 
15 Mclnnes IB, Schett G. The pathogenesis of rheumatoid arthritis. N Engl J Med 2011;365:2205-19.

16 McInnes IB, Schett G. Cytokines in the pathogenesis of rheumatoid arthritis. Nat Rev Immunol 2007;7:429-42.

17 Baka Z, Buzás E, Nagy G. Rheumatoid arthritis and smoking: putting the pieces together. Arthritis Res Ther 2009;11:238.

18 Baka Z, György B, Géher P, et al. Citrullination under physiological and pathological conditions. Joint Bone Spine 2012;79:431-6.

19 Yoshitomi H, Sakaguchi N, Kobayashi K, et al. A role for fungal \{beta\}-glucans and their receptor Dectin-1 in the induction of autoimmune arthritis in genetically susceptible mice. J Exp Med 2005;201:949-60.

20 Rehaume LM, Mondot S, Aguirre de Cárcer D, et al. Zap-70 genotype disrupts the relationship between microbiota and host, leading to spondyloarthritis and ileitis in SKG mice. Arthritis Rheumatol 2014;66:2780-92.

21 Tanaka S, Maeda S, Hashimoto M, et al. Graded attenuation of TCR signaling elicits distinct autoimmune diseases by altering thymic $T$ cell selection and regulatory $T$ cell function. J Immunol 2010;185:2295-305.

22 Pan M, Kang I, Craft J, et al. Resistance to development of collagen-induced arthritis in $\mathrm{C} 57 \mathrm{BL} / 6$ mice is due to a defect in secondary, but not in primary, immune response. J Clin Immunol 2004;24:481-91.

23 Sakaguchi S, Takahashi T, Hata H, et al. Skg mice, a new genetic model of rheumatoid arthritis. Arthritis Res Ther 2003;5:10.

24 Holmdahl R, Jansson L, Larsson E, et al. Homologous type II collagen induces chronic and progressive arthritis in mice. Arthritis Rheum 1986;29:106-13.

25 Holmdahl R, Jansson L, Gullberg D, et al. Incidence of arthritis and autoreactivity of anti-collagen antibodies after immunization of DBA/1 mice with heterologous and autologous collagen II. Clin Exp Immunol 1985;62:639-46.

26 Tong $\mathrm{D}$, Lönnblom E, Yau ACY, et al. A shared epitope of collagen type XI and type $\|$ is recognized by pathogenic antibodies in mice and humans with arthritis. Front Immunol 2018:9:451.

27 Maffia P, Brewer JM, Gracie JA, et al. Inducing experimental arthritis and breaking self-tolerance to joint-specific antigens with trackable, ovalbumin-specific T cells. $J$ Immunol 2004:173:151-6.

28 Brackertz D, Mitchell GF, Mackay IR. Antigen-Induced arthritis in mice. I. induction of arthritis in various strains of mice. Arthritis Rheum 1977;20:841-50.

29 Conigliaro P, Benson RA, Patakas A, et al. Characterization of the anticollagen antibody response in a new model of chronic polyarthritis. Arthritis Rheum 2011;63:2299-308.

30 Nickdel MB, Conigliaro P, Valesini G, et al. Dissecting the contribution of innate and antigen-specific pathways to the breach of self-tolerance observed in a murine model of arthritis. Ann Rheum Dis 2009:68:1059-66.

31 Jongbloed S, Benson RA, Mohammed B. Self-Tolerance in autoimmune arthritis 1. J Immunol 2009;182:963-8.

32 Benson RA, Patakas A, Conigliaro P, et al. Identifying the cells breaching selftolerance in autoimmunity. J Immuno/ 2010;184:6378-85

33 Vossenaar ER, van Boekel MAM, van Venrooij WJ, et al. Absence of citrullinespecific autoantibodies in animal models of autoimmunity. Arthritis Rheum 2004;50:2370-2.

34 Mclnnes IB, Schett G. Pathogenetic insights from the treatment of rheumatoid arthritis. Lancet 2017:389:2328-37.

35 Olasz K, Boldizsar F, Kis-Toth K, et al. T cell receptor (TCR) signal strength controls arthritis severity in proteoglycan-specific TCR transgenic mice. Clin Exp Immunol 2012;167:346-55.

36 Angyal A, Egelston C, Kobezda T, et al. Development of proteoglycan-induced arthritis depends on T cell-supported autoantibody production, but does not involve significant influx of T cells into the joints. Arthritis Res Ther 2010;12:R44.

37 Mandala S, Hajdu R, Bergstrom J, et al. Alteration of lymphocyte trafficking by sphingosine-1-phosphate receptor agonists. Science 2002;296:346 LP-9.

38 Matloubian M, Lo CG, Cinamon G, et al. Lymphocyte egress from thymus and peripheral lymphoid organs is dependent on S1P receptor 1. Nature 2004:427:355-60.

39 Schwab SR, Cyster JG. Finding a way out: lymphocyte egress from lymphoid organs. Nat Immunol 2007:8:1295-301.

40 Wood GS, Michie SA, Durden F, et al. Expression of class II major histocompatibility antigens by keratinocytes in cutaneous T cell lymphoma. Int J Dermatol 1994:33:346-50.

41 Sheen-Chen SM, Chou FF, Eng HL, et al. An evaluation of the prognostic significance of HLA-DR expression in axillary-node-negative breast cancer. Surgery 1994; 116:510-5

42 Svendsen $\mathrm{P}$, Andersen CB, Willcox N, et al. Tracking of proinflammatory collagenspecific $T$ cells in early and late collagen-induced arthritis in humanized mice. J Immuno/ 2004;173:7037 LP-45.

43 Mikecz K, Glant TT. Migration and homing of lymphocytes to lymphoid and synovial tissues in proteoglycan-induced murine arthritis. Arthritis Rheum 1994:37:1395-403.

44 O'Neill SK, Shlomchik MJ, Glant TT, et al. Antigen-Specific B cells are required as APCS and autoantibody-producing cells for induction of severe autoimmune arthritis. J Immuno/ 2005; 174:3781 LP-8.
45 Sun W, Meednu N, Rosenberg A, et al. B cells inhibit bone formation in rheumatoid arthritis by suppressing osteoblast differentiation. Nat Commun 2018;9:5127.

46 Miura Y, Ota S, Peterlin M, et al. A subpopulation of synovial fibroblasts leads to Osteochondrogenesis in a mouse model of chronic inflammatory rheumatoid arthritis. JBMR Plus 2019;3:e10132

47 Firestein GS, McInnes IB. Immunopathogenesis of rheumatoid arthritis. Immunity 2017;46:183-96

48 Holoshitz J. The rheumatoid arthritis HLA-DRB1 shared epitope. Rheumatology 2010;22:293-8

49 Weyand CM, Hicok KC, Conn DL, et al. The influence of HLA-DRB1 genes on disease severity in rheumatoid arthritis. Ann Intern Med 1992;117:801-6.

50 Bhayani HR, Hedrick SM. The role of polymorphic amino acids of the MHC molecule in the selection of the T cell repertoire. J Immunol 1991;146:1093-8.

51 Dyall R, Messaoudi I, Janetzki S, et al. Mhc polymorphism can enrich the $T$ cell repertoire of the species by shifts in intrathymic selection. J Immunol 2000;164:1695-8.

52 Wagner UG, Koetz K, Weyand CM, et al. Perturbation of the T cell repertoire in rheumatoid arthritis. Proc Natl Acad Sci U SA 1998;95:14447-52.

53 Ikeda Y, Masuko K, Nakai Y, et al. High frequencies of identical T cell clonotypes in synovial tissues of rheumatoid arthritis patients suggest the occurrence of common antigen-driven immune responses. Arthritis Rheum 1996:39:446-53.

54 Klarenbeek PL, de Hair MJH, Doorenspleet ME, et al. Inflamed target tissue provides a specific niche for highly expanded T-cell clones in early human autoimmune disease. Ann Rheum Dis 2012;71:1088-93.

55 Stamenkovic I, Stegagno M, Wright KA, et al. Clonal dominance among T-lymphocyte infiltrates in arthritis. Proc Nat/ Acad Sci U S A 1988:85:1179-83.

56 Waase I, Kayser C, Carlson PJ, et al. Oligoclonal T cell proliferation in patients with rheumatoid arthritis and their unaffected siblings. Arthritis Rheum 1996;39:904-13

57 Benham H, Nel HJ, Law SC, et al. Citrullinated peptide dendritic cell immunotherapy in HLA risk genotype-positive rheumatoid arthritis patients. Sci Trans/ Med 2015; 7:290ra87 LP-290.

58 Brand DD, Whittington KB, Rosloniec EF. I-Aq and I-Ap bind and present similar antigenic peptides despite differing in their ability to mediate susceptibility to autoimmune arthritis. Autoimmunity 2001:34:133-45.

59 Cantaert T, Brouard S, Thurlings RM, et al. Alterations of the synovial T cell repertoire in anti-citrullinated protein antibody-positive rheumatoid arthritis. Arthritis Rheum 2009;60:1944-56.

60 Kato T, Kurokawa M, Masuko-Hongo K, et al. T cell clonality in synovial fluid of a patient with rheumatoid arthritis: persistent but fluctuant oligoclonal T cell expansions. J Immunol 1997;159:5143-9.

61 VanderBorght A, Geusens P, Vandevyver C, et al. Skewed T-cell receptor variable gene usage in the synovium of early and chronic rheumatoid arthritis patients and persistence of clonally expanded T cells in a chronic patient. Rheumatology 2000;39:1189-201.

62 Pierer M, Rossol M, Kaltenhäuser $\mathrm{S}$, et al. Clonal expansions in selected TCR bv families of rheumatoid arthritis patients are reduced by treatment with the TNFo inhibitors etanercept and infliximab. Rheumatol Int 2011:31:1023-9.

63 Monserrat J, Bohórquez C, Gómez Lahoz AM, et al. The abnormal CD4+T lymphocyte subset distribution and Vbeta repertoire in new-onset rheumatoid arthritis can be modulated by methotrexate Treament. Cells 2019;8:871.

64 Bäcklund J, Li C, Jansson E, et al. C57BI/6 mice need MHC class II AQ to develop collagen-induced arthritis dependent on autoreactive T cells. Ann Rheum Dis 2013:72:1225-32

65 Bäcklund J, Nandakumar KS, Bockermann R, et al. Genetic control of tolerance to type II collagen and development of arthritis in an autologous collagen-induced arthritis model. J Immunol 2003:171:3493-9.

$66 \mathrm{He}$ X, Rosloniec EF, Myers LK, et al. T cell receptors recognizing type II collagen in HLA-DR-transgenic mice characterized by highly restricted $V$ beta usage. Arthritis Rheum 2004;50:1996-2004

67 Nabozny GH, Bull MJ, Hanson J, et al. Collagen-Induced arthritis in T cell receptor V beta congenic B10.Q mice. J Exp Med 1994;180:517-24.

68 Osman N, Lazarovits Al, Crumpton MJ. Physical association of CD5 and the T cell receptor/CD3 antigen complex on the surface of human T lymphocytes. Eur Immunol 1993;23:1173-6.

69 Chiocchia G, Boissier MC, Fournier C. Therapy against murine collageninduced arthritis with $\mathrm{T}$ cell receptor $\mathrm{V}$ beta-specific antibodies. Eur J Immunol 1991:21:2899-905.

70 Qian Z, Latham KA, Whittington KB, et al. An Autoantigen-Specific, highly restricted T cell repertoire infiltrates the arthritic joints of mice in an HLA-DR1 humanized mouse model of autoimmune arthritis. J Immunol 2010;185:110-8.

71 Al Khabouri S, Benson RA, Prendergast CT, et al. TCR $\beta$ sequencing reveals spatial and temporal evolution of clonal CD4 T cell responses in a breach of tolerance model of inflammatory arthritis. Front Immunol 2021;12:1399.

72 Hasegawa H, Matsumoto T. Mechanisms of Tolerance Induction by Dendritic Cells In Vivo. Front Immunol 2018;9:350.

73 Domogalla MP, Rostan PV, Raker VK, et al. Tolerance through education: how tolerogenic dendritic cells shape immunity. Front Immunol 2017;8:1764. 
74 Kim SH, Kim S, Oligino TJ, et al. Effective treatment of established mouse collageninduced arthritis by systemic administration of dendritic cells genetically modified to express FasL. Mol Ther 2002;6:584-90.

75 Stoop JN, Harry RA, von Delwig A, et al. Therapeutic effect of tolerogenic dendritic cells in established collagen-induced arthritis is associated with a reduction in Th17 responses. Arthritis Rheum 2010;62:3656-65.

76 Lim D-S, Kang M-S, Jeong J-A, et al. Semi-Mature DC are immunogenic and not tolerogenic when inoculated at a high dose in collagen-induced arthritis mice. Eur $J$ Immunol 2009;39:1334-43

77 Martin E, Capini C, Duggan E, et al. Antigen-Specific suppression of established arthritis in mice by dendritic cells deficient in NF-kappaB. Arthritis Rheum 2007:56:2255-66.

78 Schinnerling K, Rosas C, Soto L, et al. Humanized mouse models of rheumatoid arthritis for studies on immunopathogenesis and preclinical testing of cell-based therapies. Front Immunol 2019;10:203.

79 Vignali DA, Moreno J, Schiller D, et al. Species-Specific binding of CD4 to the beta 2 domain of major histocompatibility complex class II molecules. J Exp Med 1992;175:925-32.

80 Albani S, Koffeman EC, Prakken B. Induction of immune tolerance in the treatment of rheumatoid arthritis. Nat Rev Rheumatol 2011;7:272-81.

81 Pearce SHS, Dayan C, Wraith DC, et al. Antigen-Specific immunotherapy with thyrotropin receptor peptides in Graves' hyperthyroidism: a phase I study. Thyroid 2019;29:1003-11.

82 Juryńczyk M, Walczak A, Jurewicz $A$, et al. Immune regulation of multiple sclerosis by transdermally applied myelin peptides. Ann Neurol 2010;68:593-601.

83 Walczak A, Siger $\mathrm{M}$, Ciach A, et al. Transdermal application of myelin peptides in multiple sclerosis treatment. JAMA Neurol 2013;70:1105-9.

84 Sercarz EE, Lehmann PV, Ametani A, et al. Dominance and crypticity of T cell antigenic determinants. Annu Rev Immunol 1993;11:729-66.

85 Streeter HB, Rigden R, Martin KF, et al. Preclinical development and first-inhuman study of ATX-MS-1467 for immunotherapy of MS. Neurol Neuroimmunol Neuroinflamm 2015;2:e93.

86 Clemente-Casares X, Blanco J, Ambalavanan P, et al. Expanding antigen-specific regulatory networks to treat autoimmunity. Nature 2016;530:434-40.

87 Jansson L, Vrolix K, Jahraus A, et al. Immunotherapy with Apitopes blocks the immune response to TSH receptor in HLA-DR transgenic mice. Endocrinology 2018:159:3446-57.

88 Madsen LS, Andersson EC, Jansson L, et al. A humanized model for multiple sclerosis using HLA-DR2 and a human T-cell receptor. Nat Genet 1999;23:343-7.

89 De Souza ALS, Rudin S, Chang R, et al. ATX-MS-1467 Induces Long-Term Tolerance to Myelin Basic Protein in (DR2 $\times$ Ob1)F1 Mice by Induction of IL-10-Secreting iTregs. Neurol Ther 2018;7:103-28.

90 Anderton SM, Viner NJ, Matharu P, et al. Influence of a dominant cryptic epitope on autoimmune T cell tolerance. Nat Immunol 2002;3:175-81.

91 Burton BR, Britton GJ, Fang $H$, et al. Sequential transcriptional changes dictate safe and effective antigen-specific immunotherapy. Nat Commun 2014;5:4741.

92 Rosado-Sánchez I, Levings MK. Building a CAR-Treg: going from the basic to the luxury model. Cell Immunol 2020;358:104220.

93 Zhang Q, Lu W, Liang C-L, et al. Chimeric antigen receptor (CAR) Treg: a promising approach to inducing immunological tolerance. Front Immunol 2018:9:2359.

94 Sadelain M. Cd19 CAR T cells. Cell 2017;171:1471

95 Raffin C, Zhou Y, Piccoli L. Development of citrullinated-vimentin-specific CAR for targeting Tregs to treat autoimmune rheumatoid arthritis. J Immunol 2018;200:176.17 LP-176.17.

96 Chan JD, von Scheidt B, Zeng B, et al. Enhancing chimeric antigen receptor T-cell immunotherapy against cancer using a nanoemulsion-based vaccine targeting crosspresenting dendritic cells. Clin Trans/ Immunology 2020;9:e1157

97 Nagler-Anderson C, Bober LA, Robinson ME, et al. Suppression of type II collageninduced arthritis by intragastric administration of soluble type II collagen. Proc Natl Acad Sci U S A 1986;83:7443 LP-6.

98 Thompson HS, Staines NA. Gastric administration of type II collagen delays the onset and severity of collagen-induced arthritis in rats. Clin Exp Immunol 1986;64:581-6.
99 Barnett ML, Kremer JM, St Clair EW, et al. Treatment of rheumatoid arthritis with oral type II collagen. Results of a multicenter, double-blind, placebo-controlled trial. Arthritis Rheum 1998;41:290-7.

100 McKown KM, Carbone LD, Kaplan SB, et al. Lack of efficacy of oral bovine type II collagen added to existing therapy in rheumatoid arthritis. Arthritis Rheum 1999;42:1204-8.

101 Wei W, Zhang L-L, Xu J-H, et al. A multicenter, double-blind, randomized, controlled phase III clinical trial of chicken type II collagen in rheumatoid arthritis. Arthritis Res Ther 2009;11:R180.

102 Weiner HL, da Cunha AP, Quintana F, et al. Oral tolerance. Immunol Rev 2011:241:241-59.

103 Kollias G, Papadaki P, Apparailly F, et al. Animal models for arthritis: innovative tools for prevention and treatment. Ann Rheum Dis 2011;70:1357-62.

104 Doan T, McNally A, Thomas R, et al. Steady-State dendritic cells continuously inactivate $\mathrm{T}$ cells that escape thymic negative selection. Immunol Cell Biol 2009;87:615-22.

105 Capini C, Jaturanpinyo M, Chang H-I, et al. Antigen-Specific suppression of inflammatory arthritis using liposomes. J Immunol 2009;182:3556-65.

106 Galea R, Nel HJ, Talekar M, et al. PD-L1- and calcitriol-dependent liposomal antigenspecific regulation of systemic inflammatory autoimmune disease. JCI Insight 2019;4. doi:10.1172/jci.insight.126025. [Epub ahead of print: 1909 2019].

107 Korganow AS, Ji H, Mangialaio S, et al. From systemic T cell self-reactivity to organspecific autoimmune disease via immunoglobulins. Immunity 1999;10:451-61.

108 Plock JA, Schnider JT, Solari MG, et al. Perspectives on the use of mesenchymal stem cells in vascularized composite allotransplantation. Front Immunol 2013;4:175.

109 Morita Y, Yang J, Gupta R, et al. Dendritic cells genetically engineered to express IL-4 inhibit murine collagen-induced arthritis. J Clin Invest 2001;107:1275-84.

110 Piccirillo CA. Transcriptional and translational control of Foxp $3^{+}$regulatory T cell functional adaptation to inflammation. Curr Opin Immunol 2020;67:27-35.

111 Komatsu N, Okamoto K, Sawa S, et al. Pathogenic conversion of Foxp3+ T cells into Th17 cells in autoimmune arthritis. Nat Med 2014;20:62-8.

112 Graßhoff $\mathrm{H}$, Comdühr S, Monne LR, et al. Low-Dose IL-2 therapy in autoimmune and rheumatic diseases. Front Immunol 2021;12:902.

113 Nomoto K, Eto M, Yanaga K, et al. Interference with cyclophosphamide-induced skin allograft tolerance by cyclosporin A. J Immunol 1992;149:2668 LP-74.

114 Newberry RD, Stenson WF, Lorenz RG. Cyclooxygenase-2-Dependent arachidonic acid metabolites are essential modulators of the intestinal immune response to dietary antigen. Nat Med 1999;5:900-6.

115 Zeiser R, Nguyen VH, Beilhack A, et al. Inhibition of CD4+CD25+ regulatory T-cell function by calcineurin-dependent interleukin-2 production. Blood 2006;108:390-9.

116 Hijnen D, Haeck I, van Kraats AA, et al. Cyclosporin A reduces CD4 $4^{(+)}$CD2 $5^{(+)}$ regulatory T-cell numbers in patients with atopic dermatitis. J Allergy Clin Immunol 2009; 124:856-8.

117 Miroux C, Moralès 0 , Carpentier A, et al. Inhibitory effects of cyclosporine on human regulatory T cells in vitro. Transplant Proc 2009;41:3371-4.

118 Hintzen G, Ohl L, del Rio M-L, et al. Induction of tolerance to innocuous inhaled antigen relies on a CCR7-dependent dendritic cell-mediated antigen transport to the bronchial lymph node. J Immunol 2006:177:7346-54.

119 Worbs T, Bode U, Yan S, et al. Oral tolerance originates in the intestinal immune system and relies on antigen carriage by dendritic cells. J Exp Med 2006;203:519-27.

120 Garrod KR, Chang CK, Liu F-C, et al. Targeted lymphoid homing of dendritic cells is required for prolongation of allograft survival. J Immunol 2006;177:863-8.

121 Li C, Jiang P, Wei S, et al. Regulatory T cells in tumor microenvironment: new mechanisms, potential therapeutic strategies and future prospects. Mol Cancer 2020;19:116

122 Willekens B, Presas-Rodríguez S, Mansilla MJ, et al. Tolerogenic dendritic cell-based treatment for multiple sclerosis (MS): a harmonised study protocol for two phase I clinical trials comparing intradermal and intranodal cell administration. BMJ Open 2019;9:e030309.

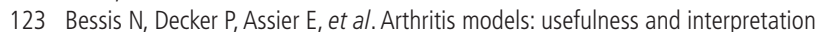
Semin Immunopathol 2017;39:469-86. 